\title{
Postoperative Acute Exacerbation of IPF after Lung Resection for Primary Lung Cancer
}

\author{
Atsushi Watanabe, Nobuyoshi Kawaharada, and Tetsuya Higami \\ Department of Thoracic and Cardiovascular Surgery, Sapporo Medical University School of Medicine, South 1, \\ West 16, Chuo-ku, Sapporo 060-8543, Japan \\ Correspondence should be addressed to Atsushi Watanabe, atsushiw@sapmed.ac.jp
}

Received 31 August 2010; Accepted 9 January 2011

Academic Editor: Aldo T. Iacono

Copyright (C) 2011 Atsushi Watanabe et al. This is an open access article distributed under the Creative Commons Attribution License, which permits unrestricted use, distribution, and reproduction in any medium, provided the original work is properly cited.

\begin{abstract}
Idiopathic pulmonary fibrosis (IPF) is characterized by slowly progressive respiratory dysfunction. Nevertheless, some IPF patients experience acute exacerbations generally characterized by suddenly worsening and fatal respiratory failure with new lung opacities and pathological lesions of diffuse alveolar damage. Acute exacerbation of idiopathic pulmonary fibrosis (AEIPF) is a fatal disorder defined by rapid deterioration of IPF. The condition sometimes occurs in patients who underwent lung resection for primary lung cancer in the acute and subacute postoperative phases. The exact etiology and pathogenesis remain unknown, but the condition is characterized by diffuse alveolar damage superimposed on a background of IPF that probably occurs as a result of a massive lung injury due to some unknown factors. This systematic review shows that the outcome, however, is poor, with postoperative mortality ranging from $33.3 \%$ to $100 \%$. In this paper, the etiology, risk factors, pathogenesis, therapy, prognosis, and predictors of postoperative AEIPF are described.
\end{abstract}

\section{Introduction and Background}

The incidence of lung cancer in patients with idiopathic pulmonary fibrosis (IPF) is higher than the general population, with relative risks reported to be from 7 to $14 \%$ [1-3] IPF is usually a gradually progressive but an ultimately fatal disease. Although the disease is chronic in nature, abrupt worsening can occur in some patients. This condition was first introduced by Kondoh and Saiki [4] and was then called acute exacerbation of IPF (AEIPF). The prognosis of AEIPF is usually considered to be grave, but it has been reported that some patients show improvement following corticosteroid therapy. It still remains uncertain what causes such an acute exacerbation, and appropriate therapy for this condition has not been established. In the survey by the Japanese association of thoracic surgery, 1036 of 27881 patients who underwent pulmonary resection for primary lung cancer during the year 2008 had interstitial pneumonia as a preoperative comorbidity. Although the hospital mortality was about $0.9 \%$ (248 patients died after the operation), 63 of 248 patients (25.4\%) died of interstitial pneumonia, including AEIP. We are focused on the current knowledge of AEIPF and what causes the exacerbation after pulmonary resection for nonsmall cell lung cancer (NSCLC).

\section{The Content}

2.1. Diagnostic Criteria for Acute Exacerbation of IPF. The acute exacerbation of IPF (AEIPF) is characterized by diffuse and rapid alveolar damage superimposed on a background of IPF that probably occurs as a result of a massive lung injury due to some unknown etiologic agent. The definition of AEIPF was first described by Yoshimura et al. [5]. The characteristics include (1) intensified dyspnea, (2) increase in the interstitial shadow on chest radiograph, (3) increase in fine crackles on auscultation, (4) elevation of serum lactate dehydrogenase, and (5) decrease in arterial oxygen tension of more than $10 \mathrm{~mm} \mathrm{Hg}$ under similar condition. After then, some diagnostic criteria have been described [6-11]. In the clinical field and the surgical field, the definition described by Hyzy has been generally applied (Table 1). 
TABLE 1: Definition of acute exacerbation of IPF described by Hyzy et al. [10].

Previous or concurrent diagnosis of IPF*

Unexplained worsening or development of dyspnea within $30 \mathrm{~d}$

High-resolution CT scan with new bilateral ground-glass

abnormality and/or consolidation superimposed on a

background reticular or honeycomb pattern consistent with a UIP pattern ${ }^{\dagger}$

Worsening hypoxemia from a known baseline arterial blood gas $^{\ddagger}$

No evidence of pulmonary infection by endotracheal aspiration or BAL

Exclusion of alternative causes, including

left heart failure

pulmonary embolism

identifiable cause of acute lung injury ${ }^{\S}$

* This criterion can be met by the presence of radiologic and/or histopathologic changes consistent with a UIP pattern if a diagnosis of IPF has not been previously established by American Thoracic Society/European Respiratory Society criteria.

${ }^{\dagger}$ Current high-resolution CT scan is acceptable without prior highresolution CT scan for comparison if none is available.

‡Includes evaluation for common bacterial organisms and viral pathogens.

$\S$ Causes of lung injury include sepsis, aspiration, trauma, transfusion of blood products, pulmonary contusion, fat embolization, drug toxicity, acute pancreatitis, inhalational injury, and cardiopulmonary bypass.

Especially, bacterial pneumonia must be distinguished from AEIPF. Pneumonia is diagnosed by the presence of new and/or progressive pulmonary infiltrates on chest radiography plus two or more of the following criteria: fever $\left(38^{\circ} \mathrm{C}\right)$, leukocytosis $(12 \times 109 / \mathrm{L})$, purulent sputum, or isolation of pathogen in respiratory secretions. If necessary, endotracheal aspiration or BA is performed.

2.2. Etiology. Specific factors causing AEIPF have not been elucidated. However, some cases of AEIPF have occurred after lung resection or biopsy [13, 21]. Kondoh et al. [22] observed that postbiopsy exacerbation occurred in $2.1 \%$ of 236 consecutive patients who underwent surgical biopsy for diffuse lung disease. AEIPF appear to occur at any time during the course of disease and may be the presenting manifestation for some patients. Importantly, the risk of an exacerbation does not appear to be linked to the level of pulmonary function [23]; although in one prospective series, patients with lower forced vital capacity had more total and respiratory-related hospitalizations during subsequent followup [24]. There is no clear association with age or smoking history, but acute exacerbations seem to be more common in men.

2.3. Pathology. AEIPF is an acute insult to the lung over a background of IPF. According to some autopsy studies [2527], there was a wide distribution in the extent of fibroblastic foci but not seen in fibrotic nonspecific interstitial pneumonia. Fibroblastic foci were distinguished from buds of intraalveolar organization seen in the organizing phase of diffuse alveolar damage by their immediate adjacency to areas of established fibrosis and their presence away from areas of established diffuse alveolar damage. Areas showing honeycomb changes contained aggregates of abundant neutrophils within the air spaces; however, staining for organisms was uniformly negative in these areas and the results for tissue taken for culture at autopsy also were negative.

2.4. Incidence and Mortality of Postoperative AEIPF. According to the annual report by the Japanese association of thoracic surgery [28], 1036 of 27881 patients (3.7\%) who underwent pulmonary resection for primary lung cancer during the year 2008 had interstitial pneumonia as a preoperative comorbidity. Although the whole hospital mortality was about $0.9 \%$ (248 patients died after the operation), 63 patients $(25.4 \%)$ died of interstitial pneumonia, including AEIPF. Additionally, 157 patients suffered from AEIPF during hospitalization.

Six (15.0\%) of 40 patients with IPF had AEIPF after the operation and 5 of the six died with respiratory failure at POD 47 on average (range 17-95 days) [20]. We reported that $4(7.1 \%)$ of these 56 patients developed postoperative AEIPF, and all of them died of respiratory failure within 42 days after the operation despite immunosuppression with pulse doses of methylprednisolone [18]. The rate of occurrence and mortality of postoperative AEIPF in the literatures $[12,14,16,17]$ are summarized in Table 2. The incidence of postoperative AEIPF ranged from $0 \%$ to $20.8 \%$, mortality after pulmonary resection from $8.3 \%$ to $22.9 \%$, and mortality after occurrence of AEIPF from $37.5 \%$ to $100 \%$. On the other hand, AEIPF may occur after any medical treatment for cancer. Minegishi et al. reported that the incidence and mortality after each anti-lung cancer therapy, including pulmonary resection, were $20 \%$ and $14 \%$ for chemotherapy $(n=50), 42.9 \%$ and $14.3 \%$ for chemoradiotherapy $(n=7), 16.7 \%$ and $16.7 \%$ for radiation therapy $(n=6), 22.9 \%$ and $8.9 \%$ for pulmonary resection $(n=35)$, and $31.3 \%$ and $31.3 \%$ for best supportive care $(n=32)$ [19], respectively.

2.5. Risks of Postoperative Exacerbation of IPF. Kumar et al. [13] reported that postoperative ARDS was associated with lower preoperative levels of carbon monoxide diffusion capacity corrected for alveolar volume $\left(K_{\mathrm{CO}}\right)$, lower preoperative DLCO levels, and higher preoperative composite physiological index $[29](\mathrm{CPI}=91.0-(0.65 \times \%$ predicted DLCO $)-(0.53 \times \%$ predicted FVC $)+(0.34 \times \%$ predicted FEV1)). A preoperative CPI score $>40$ was associated with a $50 \%$ chance of developing postoperative lung injury. The occurrence of ARDS was not related to demographic features, smoking history, other pulmonary function variables, presence of preoperative dyspnea, histologically established diagnosis of pulmonary fibrosis before pulmonary resection, stage of nonsmall cell lung cancer, or pattern of inflammation.

$\%$ vital capacity (VC) $(<80.6 \%)$ and LDH level $(\geq 241 \mathrm{IU} / \mathrm{L})$ achieved a complete classification of patients with AEIPF from those without AEIPF [28]. The other researchers reported that postoperative AEIP were associated 
TABLE 2: The incidence and mortality of postoperative acute exacerbation of IPF.

\begin{tabular}{lllllllll}
\hline Author & $\begin{array}{l}\text { Published } \\
\text { year }\end{array}$ & $\begin{array}{l}\text { No. of } \\
\text { AEIPF }\end{array}$ & $\begin{array}{l}\text { Incidence of } \\
\text { AEIPF (\%) }\end{array}$ & $\begin{array}{l}\text { Death of } \\
\text { AEIPF }\end{array}$ & Mortality & $\begin{array}{l}\text { Mortality } \\
\text { after AEIPF }\end{array}$ & $\begin{array}{l}\text { No. of IPF } \\
\text { Pts }\end{array}$ & $\begin{array}{l}\text { Total no. of } \\
\text { Pts }\end{array}$ \\
\hline Fujimoto et al. [12] & 2003 & 0 & 0 & 0 & 0 & NA & 21 & NA \\
Kumar et al. [13] & 2003 & 5 (ARDS) & 20.8 & 4 & 16.7 & 80 & 24 & 988 \\
Chiyo et al. [14] & 2003 & 9 & 25 & 3 & 8.3 & 33.3 & 36 & 931 \\
Koizumi et al. [15] & 2004 & 7 & 14.9 & 6 & 12.8 & 85.7 & 47 & 1103 \\
Okamoto et al. [16] & 2004 & 4 & 20 & 3 & 20 & 75 & 20 & NA \\
Kushibe et al. [17] & 2007 & 4 & 12.1 & 4 & 18.2 & 100 & 33 & 1066 \\
Watanabe et al. [18] & 2008 & 4 & 7.4 & 4 & 7.4 & 100 & 54 & 870 \\
Minegishi et al. [19] & 2009 & 8 & 8.6 & 3 & 22.9 & 37.5 & 35 & NA \\
Shintani et al. [20] & 2010 & 6 & 15 & 5 & 12.5 & 83.3 & 40 & 1256 \\
\hline
\end{tabular}

AEIPF: acute exacerbation of idiopathic pulmonary fibrosis, NA: not available, Pts: patients, ARDS: acute respiratory distress syndrome.

with \%VC $<80 \%[16,17]$, low DLCO [16], and \%TLC $<$ $95 \%[29]$.

The incidence of postoperative complications associated with video-assisted thoracoscopic surgery (VATS) seemed to be low although the use of VATS could not prevent AE of usual interstitial pneumonia (UIP) [15].

On the other hand, our study demonstrated no observed predictive risks of postoperative AEIPF after lung resection for primary lung cancer [18]. The risks, which are reported in the literatures, are shown on Table 3. However, these reports are from the study of a single institution and the number of patients is too small for statistical analysis of the risks. The aforementioned pose as limitations of the study. In the Japanese association of chest surgery, a multi-institutional Study of the postoperative exacerbation of IPF is currently being conducted.

2.6. Strategy for Decreasing the Incidence of Postoperative $A E I P F$. Surgical approaches, such as conventional thoracotomy, muscle sparing thoracotomy, and video-assisted thoracoscopic surgery have no effect on the occurrence of postoperative AEIPF [30]. Some unknown or potential etiologic agents of AEIPF must be induced by pulmonary resection or some factors related to pulmonary resection, such as selective lung ventilation and manipulation of the ipsilateral lung. If oxygen radical toxicity can be associated to the occurrence of AEIPF, Misthos et al. [31] recently reported this interesting theory. The authors revealed the following results: (1) lung re-expansion after one-lung ventilation (OLV) provoked severe oxidative stress; (2) the degree of generated oxygen-derived free radicals was associated with the duration of OLV; (3) patients with lung cancer had a higher production of oxygen-derived free radicals than the normal population; (4) tumor resection removes a large oxidative burden from the organism; (5) mechanical ventilation and surgical trauma are weak free radical generators; (6) manipulated lung tissue is also a source of oxygenderived free radicals, not only intraoperatively but also for several hours later. These results indicate that shortening the duration of OLV and avoiding manipulation of lung tissue may inhibit the occurrence of AEIPF resulting from oxygenderived free radicals.
No drug has been established to decrease the incidence of AEIPF. Few studies reported that steroid, pirfenidone [32], and anticoagulants [33] reduce the occurrence of AEIPF in patients with IPF. However, regarding postoperative AEIPF, so far, no researchers presented a drug that can prevent or decrease the occurrence of postoperative AEIPF. Thus, thoracic surgeons use some drugs reported to reduce the incidence of AEIPF or slow the deterioration of IPF, such as macrolides [34, 35], $\mathrm{N}$-acetylcysteine [36-39], proteinase inhibitor [40,41], and pirfenidone [42, 43]. The effect of these drugs on decreasing the incidence of postoperative AEIPF remains unclear; therefore, multi-institutional randomized controlled study should be planned in order to determine the effect of these drugs.

2.7. Treatment of Postoperative AEIPF. In the absence of any effective therapeutic regimens, postoperative AEIPF seems to be a fatal problem. Although most patients with nonpostoperative AEIPF have been treated with regimens that include high-dose corticosteroids [44], the vast majority shows only partial and temporary improvement. In postoperative AEIPF, the situation is similar. Some investigators have even suggested that mechanical ventilation does not benefit IPF patients presenting with acute respiratory failure [45]. Yokoyama and associates [46] suggest that noninvasive ventilation can be a possible option for the management of acute respiratory failure in patients with AEIPF.

Some studies reported that immunosuppressive agents, such as Cyclosporine A [47-49] and cyclophosphamide [50], improve the prognosis of AEIPF. However, Okamoto et al. [16] reported that patients with AEIPF under methylprednisolone pulse therapy in combination with cyclophosphamide or cyclosporine A did not significantly improve the outcome of AEIPF. In conclusion, there is little evidence that currently accepted treatments are effective in AEIPF, and further studies are needed to clarify the pathogenesis and contribute to the prevention of AEIPF. On the other hand, there are some reports on the effect of hemoperfusion $[51,52]$ on AEIPF. The study by Seo et al. showed that six patients with AEIPF underwent polymyxin B-immobilized fiber column (PMX) hemoperfusion treatment. In four of 
TABLE 3: The risk predictors of the postoperative acute exacerbation.

\begin{tabular}{lll}
\hline Author & Published year & Risk predictors of postoperative AEIPF \\
\hline Kumar et al. [13] & 2003 & Low \%DLco (AEIPF+ versus AEIPF-; 48\% versus 58\%), Low \% $K_{\mathrm{CO}}(58 \%$ versus \\
Koizumi et al. [15] & 2004 & PS $>$, High CPI $(44$ versus 33$)$ \\
Okamoto et al. [16] & 2004 & $\% \mathrm{FC}<80$, LowDLco (value is not described) \\
Kushibe et al. [17] & 2007 & $\% \mathrm{VC}<80 \%$ \\
Watanabe et al. [18] & 2008 & None \\
Shintani et al. [20] & 2010 & $\% \mathrm{VC}(<80.6 \%)$ and LDH $(\geq 241 \mathrm{IU} / \mathrm{L})$ \\
\hline
\end{tabular}

$K_{\mathrm{CO}}$ : levels of carbon monoxide diffusion capacity corrected for alveolar volume;

CPI: composite physiological index; PS: performance status; TLC: total lung capacity;

DLCO: diffusing capacity of the lung for carbon monoxide.

six patients, alveolar-arterial difference of oxygen, serum KL6 , and lactate dehydrogenase level were improved after PMX treatment. These four patients were successfully weaned from mechanical ventilation and survived more than 30 days after the initial PMX treatment. However, this study involved a small number of patients and/or the absence of randomization. A randomized controlled study will be more factual.

\section{Conclusion and Future Directions}

AEIPF frequently occurs after lung resection for lung cancer with fatal outcome. Nowadays, there is no definitive treatment guidelines and effective prevention on the occurrence of AEIPF. A randomized study with statistically adequate number of patients is required to identify the etiology, risk factors, prognostic markers, and effective treatments.

\section{References}

[1] R. J. Panos, R. L. Mortenson, S. A. Niccoli, and T. E. King Jr., "Clinical deterioration in patients iwth idiopathic pulmonary fibrosis: causes and assessment," American Journal of Medicine, vol. 88, no. 4, pp. 396-404, 1990.

[2] J. Park, D. S. Kim, T. S. Shim et al., "Lung cancer in patients with idiopathic pulmonary fibrosis," European Respiratory Journal, vol. 17, no. 6, pp. 1216-1219, 2001.

[3] American Thoracic Society, "European respiratory society international multidisciplinary concensus classification of the idiopathic interstitial pneumonias," American Journal of Respiratory and Critical Care Medicine, vol. 165, pp. 277-304, 2002.

[4] A. Kondo and S. Saiki, "Acute exacerbation in idiopathic interstitial pneumonia (IIP)," in Interstitial Pneumonia of Unknown Etiology, M. Harasawa, Y. Fukuchi, and H. Morinari, Eds., vol. 27 of Intractable Diseases Research Foundation Publication, pp. 33-42, University of Tokyo Press, Tokyo, Japan, 1989.

[5] K. Yoshimura, T. Nakatani, and Y. Nakamori, "Acute exacerbation in idiopathic interstitial pneumonia," Nihon Kokyuki Gakkai Zasshi, vol. 22, no. 11, pp. 1012-1020, 1984 (Japanese).

[6] M. Akira, H. Hamada, M. Sakatani, C. Kobayashi, M. Nishioka, and S. Yamamoto, "CT findings during phase of accelerated deterioration in patients with idiopathic pulmonary fibrosis," American Journal of Roentgenology, vol. 168, no. 1, pp. 79-83, 1997.

[7] Y. Kondoh, H. Taniguchi, Y. Kawabata, T. Yokoi, K. Suzuki, and K. Takagi, "Acute exacerbation in idiopathic pulmonary fibrosis: analysis of clinical and pathologic findings in three cases," Chest, vol. 103, no. 6, pp. 1808-1812, 1993.

[8] J. J. Swigris and K. K. Brown, "Acute interstitial pneumonia and acute exacerbations of idiopathic pulmonary fibrosis," Seminars in Respiratory and Critical Care Medicine, vol. 27, no. 6, pp. 659-667, 2006.

[9] D. S. Kim, H. R. Collard, and T. E. King, "Classification and natural history of the idiopathic interstitial pneumonias," Proceedings of the American Thoracic Society, vol. 3, no. 4, pp. 285-292, 2006.

[10] R. Hyzy, S. Huang, J. Myers, K. Flaherty, and F. Martinez, "Acute exacerbation of idiopathic pulmonary fibrosis," Chest, vol. 132, no. 5, pp. 1652-1658, 2007.

[11] H. R. Collard, B. B. Moore, K. R. Flaherty et al., "Acute exacerbations of idiopathic pulmonary fibrosis," American Journal of Respiratory and Critical Care Medicine, vol. 176, no. 7, pp. 636-643, 2007.

[12] T. Fujimoto, T. Okazaki, T. Matsukura et al., "Operation for lung cancer in patients with idiopathic pulmonary fibrosis: surgical contraindication?" Annals of Thoracic Surgery, vol. 76, no. 5, pp. 1674-1678, 2003.

[13] P. Kumar, P. Goldstraw, K. Yamada et al., "Pulmonary fibrosis and lung cancer: risk and benefit analysis of pulmonary resection," Journal of Thoracic and Cardiovascular Surgery, vol. 125, no. 6, pp. 1321-1327, 2003.

[14] M. Chiyo, Y. Sekine, T. Iwata et al., "Impact of interstitial lung disease on surgical morbidity and mortality for lung cancer: analyses of short-term and long-term outcomes," Journal of Thoracic and Cardiovascular Surgery, vol. 126, no. 4, pp. 11411146, 2003.

[15] K. Koizumi, T. Hirata, K. Hirai et al., "Surgical treatment of lung cancer combined with interstitial pneumonia: the effect of surgical approach on postoperative acute exacerbation," Annals of Thoracic and Cardiovascular Surgery, vol. 10, no. 6, pp. 340-346, 2004.

[16] T. Okamoto, M. Gotoh, D. Masuya et al., "Clinical analysis of interstitial pneumonia after surgery for lung cancer," Japanese Journal of Thoracic and Cardiovascular Surgery, vol. 52, no. 7, pp. 323-329, 2004.

[17] K. Kushibe, T. Kawaguchi, M. Takahama, M. Kimura, T. Tojo, and S. Taniguchi, "Operative indications for lung cancer with idiopathic pulmonary fibrosis," Thoracic and Cardiovascular Surgeon, vol. 55, no. 8, pp. 505-508, 2007. 
[18] A. Watanabe, T. Higami, S. Ohori, T. Koyanagi, S. Nakashima, and T. Mawatari, "Is lung cancer resection indicated in patients with idiopathic pulmonary fibrosis?" Journal of Thoracic and Cardiovascular Surgery, vol. 136, no. 5, pp. 1357$1363,2008$.

[19] Y. Minegishi, K. Takenaka, H. Mizutani et al., "Exacerbation of idiopathic interstitial pneumonias associated with lung cancer therapy," Internal Medicine, vol. 48, no. 9, pp. 665-672, 2009.

[20] Y. Shintani, M. Ohta, T. Iwasaki et al., "Predictive factors for postoperative acute exacerbation of interstitial pneumonia combined with lung cancer," General Thoracic and Cardiovascular Surgery, vol. 58, no. 4, pp. 182-185, 2010.

[21] M. Yüksel, M. O. Özyurtkan, K. Bostanci, R. Ahiskali, and N. Kodalli, "Acute exacerbation of interstitial fibrosis after pulmonary resection," Annals of Thoracic Surgery, vol. 82, no. 1, pp. 336-338, 2006.

[22] Y. Kondoh, H. Taniguchi, M. Kitaichi et al., "Acute exacerbation of interstitial pneumonia following surgical lung biopsy," Respiratory Medicine, vol. 100, no. 10, pp. 1753-1759, 2006.

[23] D. S. Kim, J. H. Park, B. K. Park, J. S. Lee, A. G. Nicholson, and T. Colby, "Acute exacerbation of idiopathic pulmonary fibrosis: frequency and clinical features," European Respiratory Journal, vol. 27, no. 1, pp. 143-150, 2006.

[24] F. J. Martinez, S. Safrin, D. Weycker et al., "The clinical course of patients with idiopathic pulmonary fibrosis," Annals of Internal Medicine, vol. 142, no. 12, pp. 963-967, 2005.

[25] A. J. Rice, A. U. Wells, D. Bouros et al., "Terminal diffuse alveolar damage in relation to interstitial pneumonias: an autopsy study," American Journal of Clinical Pathology, vol. 119, no. 5, pp. 709-714, 2003.

[26] J. G. Parambil, J. L. Myers, and J. H. Ryu, "Histopathologic features and outcome of patients with acute exacerbation of idiopathic pulmonary fibrosis undergoing surgical lung biopsy," Chest, vol. 128, no. 5, pp. 3310-3315, 2005.

[27] C. E. Daniels, E. S. Yi, and J. H. Ryu, "Autopsy findings in 42 consecutive patients with idiopathic pulmonary fibrosis," European Respiratory Journal, vol. 32, no. 1, pp. 170-174, 2008.

[28] R. Sakata, Y. Fujii, and H. Kuwano, "Thoracic and cardiovascular surgery in Japan during 2008," General Thoracic and Cardiovascular Surgery, vol. 58, no. 7, pp. 356-383, 2010.

[29] A. U. Wells, S. R. Desai, M. B. Rubens et al., "Idiopathic pulmonary fibrosis: a composite physiologic index derived from disease extent observed by computed tomography," American Journal of Respiratory and Critical Care Medicine, vol. 167, no. 7, pp. 962-969, 2003.

[30] K. Koizumi, T. Hirata, K. Hirai et al., "Surgical treatment of lung cancer combined with interstitial pneumonia: the effect of surgical approach on postoperative acute exacerbation," Annals of Thoracic and Cardiovascular Surgery, vol. 10, no. 6, pp. 340-346, 2004.

[31] P. Misthos, S. Katsaragakis, N. Milingos et al., "Postresectional pulmonary oxidative stress in lung cancer patients. The role of one-lung ventilation," European Journal of Cardio-Thoracic Surgery, vol. 27, no. 3, pp. 379-383, 2005.

[32] A. Azuma, T. Nukiwa, E. Tsuboi et al., "Double-blind, placebocontrolled trial of pirfenidone in patients with idiopathic pulmonary fibrosis," American Journal of Respiratory and Critical Care Medicine, vol. 171, no. 9, pp. 1040-1047, 2005.

[33] H. Kubo, K. Nakayama, M. Yanai et al., "Anticoagulant therapy for idiopathic pulmonary fibrosis," Chest, vol. 128, no. 3, pp. 1475-1482, 2005.

[34] A. Azuma, T. Furuta, T. Enomoto et al., "Preventive effect of erythromycin on experimental bleomycin-induced acute lung injury in rats," Thorax, vol. 53, no. 3, pp. 186-189, 1998.
[35] Y. Li, A. Azuma, S. Takahashi et al., "Fourteen-membered ring macrolides inhibit vascular cell adhesion molecule 1 messenger RNA induction and leukocyte migration: role in preventing lung injury and fibrosis in bleomycin-challenged mice," Chest, vol. 122, no. 6, pp. 2137-2145, 2002.

[36] D. M. Radomska-Leśniewska, E. Skopińska-Rózewska, E. Jankowska-Steifer et al., "N-acetylcysteine inhibits IL-8 and MMP-9 release and ICAM-1 expression by bronchoalveolar cells from interstitial lung disease patients," Pharmacological Reports, vol. 62, no. 1, pp. 131-138, 2010.

[37] J. Behr, M. Demedts, R. Buhl et al., "Lung function in idiopathic pulmonary fibrosis-extended analyses of the IFIGENIA trial," Respiratory Research, vol. 10, pp. 101-110, 2009.

[38] M. Demedts, J. Behr, R. Buhl et al., "High-dose acetylcysteine in idiopathic pulmonary fibrosis," New England Journal of Medicine, vol. 353, no. 21, pp. 2229-2242, 2005.

[39] G. W. Hunninghake, "Antioxidant therapy for idiopathic pulmonary fibrosis," New England Journal of Medicine, vol. 353, no. 21, pp. 2285-2287, 2005.

[40] Y. Taooka, A. Maeda, K. Hiyama, S. Ishioka, and M. Yamakido, "Effects of neutrophil elastase inhibitor on bleomycin-induced pulmonary fibrosis in mice," American Journal of Respiratory and Critical Care Medicine, vol. 156, no. 1, pp. 260-265, 1997.

[41] M. Nakamura, T. Ogura, N. Miyazawa et al., "Outcome of patients with acute exacerbation of idiopathic interstitial fibrosis (IPF) treated with sivelestat and the prognostic value of serum KL-6 and surfactant protein D," Nihon Kokyuki Gakkai Zasshi, vol. 45, no. 6, pp. 455-459, 2007 (Japanese).

[42] G. Raghu, W. C. Johnson, D. Lockhart, and Y. Mageto, "Treatment of idiopathic pulmonary fibrosis with a new antifibrotic agent, pirfenidone: results of a prospective, openlabel phase II study," American Journal of Respiratory and Critical Care Medicine, vol. 159, no. 4, pp. 1061-1069, 1999.

[43] H. Taniguchi, M. Ebina, Y. Kondoh et al., "Pirfenidone in idiopathic pulmonary fibrosis," European Respiratory Journal, vol. 35, no. 4, pp. 821-829, 2010.

[44] R. J. Panos, R. L. Mortenson, S. A. Niccoli, and T. E. King Jr., "Clinical deterioration in patients iwth idiopathic pulmonary fibrosis: causes and assessment," American Journal of Medicine, vol. 88, no. 4, pp. 396-404, 1990.

[45] J. B. Stern, H. Mal, O. Groussard et al., "Prognosis of patients with advanced idiopathic pulmonary fibrosis requiring mechanical ventilation for acute respiratory failure," Chest, vol. 120, no. 1, pp. 213-219, 2001.

[46] T. Yokoyama, Y. Kondoh, H. Taniguchi et al., "Noninvasive ventilation in acute exacerbation of idiopathic pulmonary fibrosis," Internal Medicine, vol. 49, no. 15, pp. 1509-1514, 2010.

[47] S. Homma, S. Sakamoto, M. Kawabata et al., "Cyclosporin treatment in steroid-resistant and acutely exacerbated interstitial pneumonia," Internal Medicine, vol. 44, no. 11, pp. 11441150, 2005.

[48] S. Sakamoto, S. Homma, A. Miyamoto, A. Kurosaki, T. Fujii, and K. Yoshimura, "Cyclosporin A in the treatment of acute exacerbation of idiopathic pulmonary fibrosis," Internal Medicine, vol. 49, no. 2, pp. 109-115, 2010.

[49] N. Inase, M. Sawada, Y. Ohtani et al., "Cyclosporin A followed by the treatment of acute exacerbation of idiopathic pulmonary fibrosis with corticosteroid," Internal Medicine, vol. 42, no. 7, pp. 565-570, 2003.

[50] V. Ambrosini, A. Cancellieri, M. Chilosi et al., "Acute exacerbation of idiopathic pulmonary fibrosis: report of a series," European Respiratory Journal, vol. 22, no. 5, pp. 821-826, 2003. 
[51] Y. Seo, S. Abe, M. Kurahara et al., "Beneficial effect of polymyxin B-immobilized fiber column (PMX) hemoperfusion treatment on acute exacerbation of idiopathic pulmonary fibrosis," Internal Medicine, vol. 45, no. 18, pp. 1033-1038, 2006.

[52] N. Enomoto, T. Suda, T. Uto et al., "Possible therapeutic effect of direct haemoperfusion with a polymyxin B immobilized fibre column (PMX-DHP) on pulmonary oxygenation in acute exacerbations of interstitial pneumonia," Respirology, vol. 13, no. 3, pp. 452-460, 2008. 


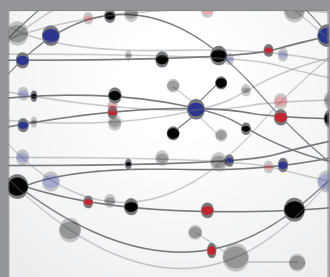

The Scientific World Journal
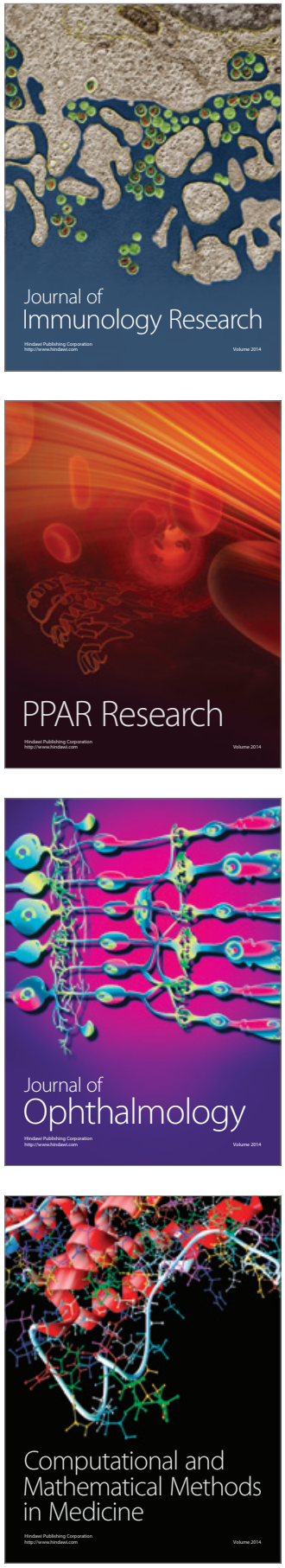

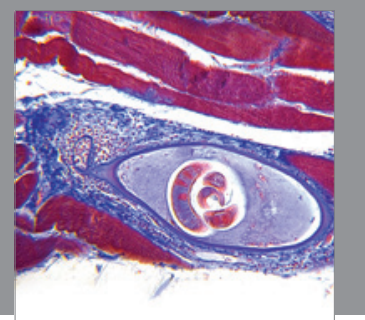

Gastroenterology

Research and Practice
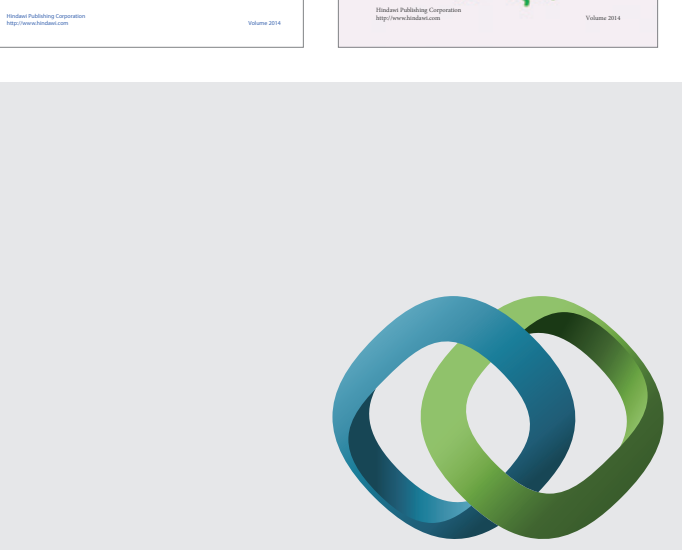

\section{Hindawi}

Submit your manuscripts at

http://www.hindawi.com
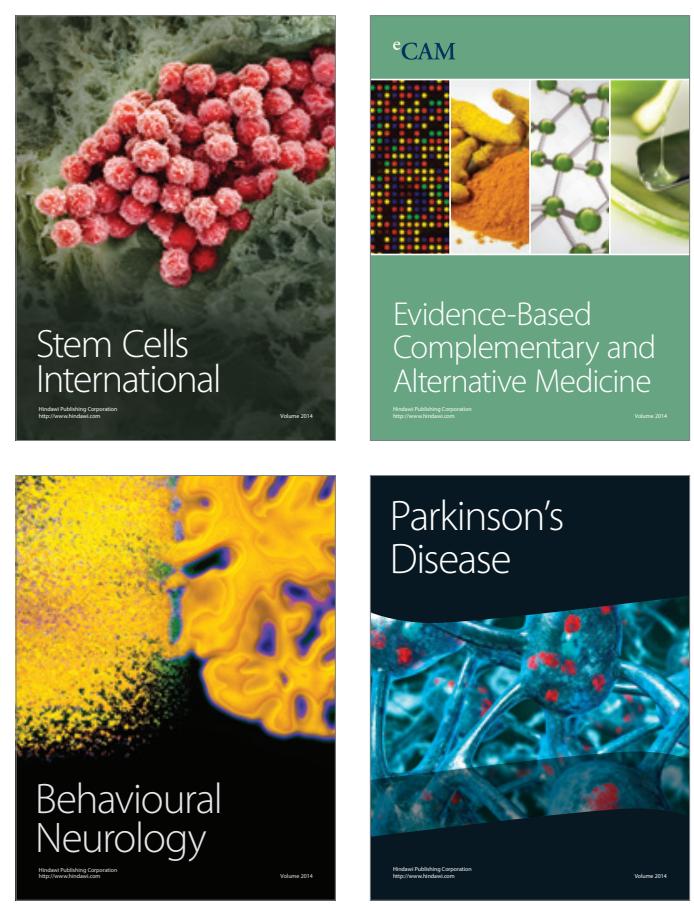

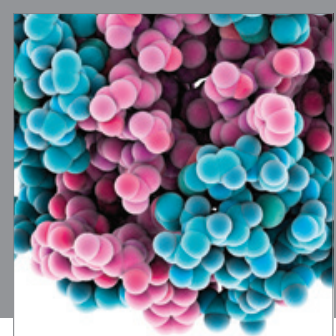

Journal of
Diabetes Research

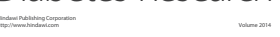

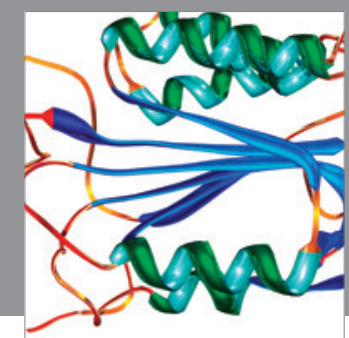

Disease Markers
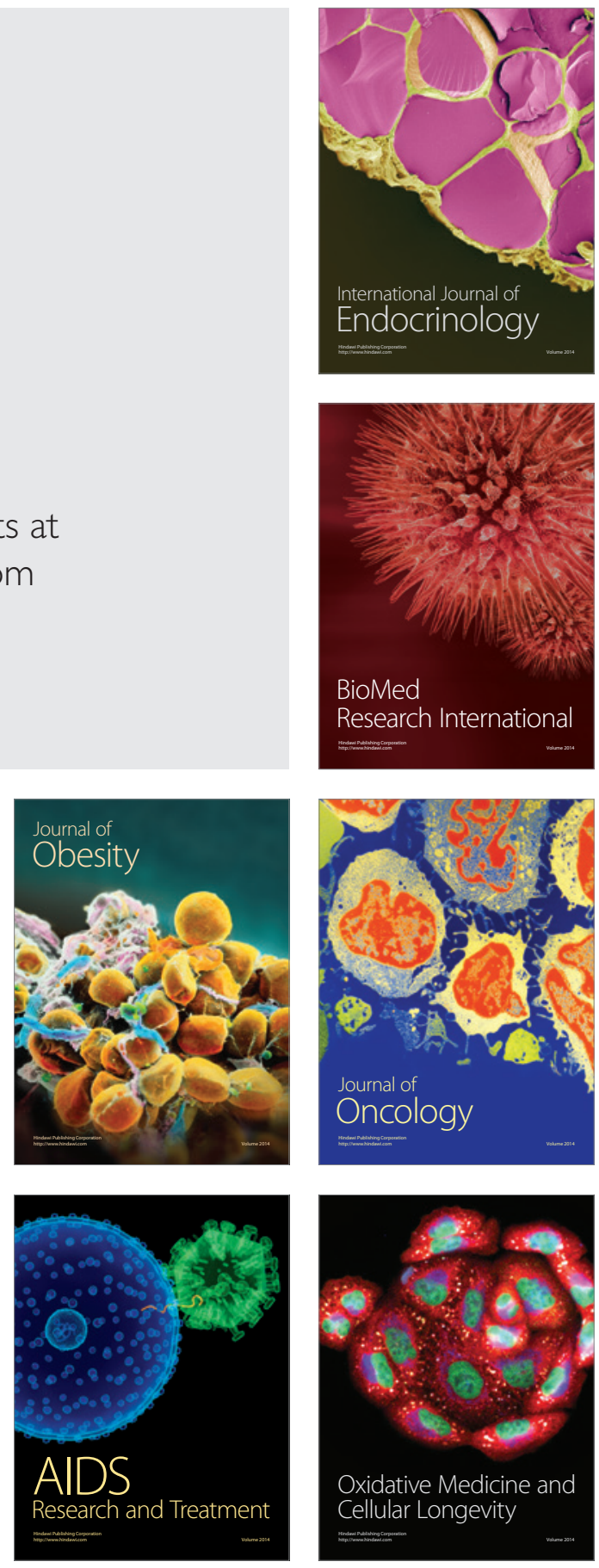